\title{
Consumer Acceptance and Expectations of a Mobile Health Application to Photograph Skin Lesions for Early Detection of Melanoma
}

\author{
Uyen Koh $^{a}$ Caitlin Horsham ${ }^{a, b} \quad$ H. Peter Soyer ${ }^{c} d \quad$ Lois J. Loescher ${ }^{\mathrm{e}}$ \\ Nicole Gillespie ${ }^{f}$ Dimitrios Vagenas ${ }^{b}$ Monika Janda ${ }^{a}$ b \\ ${ }^{a}$ Centre of Health Services Research, Faculty of Medicine, The University of Queensland, Brisbane, QLD, Australia; \\ ${ }^{b}$ School of Public Health and Social Work, Institute for Health and Biomedical Innovation, Queensland University of \\ Technology, Brisbane, QLD, Australia; ' Dermatology Research Centre, The University of Queensland, The University \\ of Queensland Diamantina Institute, Brisbane, QLD, Australia; ${ }^{\mathrm{d}}$ Dermatology Department, Princess Alexandra \\ Hospital, Brisbane, QLD, Australia; ' Colleges of Nursing and Public Health, The University of Arizona, Tucson, AZ, \\ USA; ${ }^{f}$ UQ Business School, The University of Queensland, Brisbane, QLD, Australia
}

\section{Keywords}

Telehealth · Telemedicine - Teledermatology •

Teledermoscopy · Mobile health apps · Melanoma

\begin{abstract}
Background: Mobile teledermoscopy may facilitate skin self-examinations (SSEs) and further improve monitoring and detection of melanoma. Objective: To assess consumer acceptability and expectations of a mobile health app used to: (i) instruct SSE and (ii) conduct consumer-performed mobile teledermoscopy. Methods: People aged 18 years and above were invited to participate in either (i) an online survey or (ii) focus group in Brisbane, Australia. Participants were asked about their SSE practices, mobile teledermoscopy acceptance, and app design and functionality. The online survey responses and focus group discussions were coded by two researchers who conducted thematic analysis. $\boldsymbol{R} \boldsymbol{e}$ sults: Four focus groups were held with a total of 28 participants; 88 participants completed the online survey. The mean ages of participants in the focus group and online survey were 46 and 38 years, respectively. There were more males in the focus groups $(61 \%, 17 / 28)$ compared to the online survey (19\%, 17/88). Regular SSEs were conducted by 56
\end{abstract}

(64\%) of the online survey participants. Barriers to SSE were forgetfulness (44\%), low self-perceived risk of melanoma $(25 \%)$ and low confidence in conducting SSEs (25\%). The large majority of online survey participants (95\%) would consider sending photos of their skin lesions to a medical practitioner via an app. Focus group participants reported that they would accept using mobile teledermoscopy; however, they would prefer to use it to monitor lesions between face-to-face consultations. Conclusions: Overall, participants had positive views on using mobile teledermoscopy to send images of skin lesions to a dermatologist or other medical practitioner.

๑) 2018 S. Karger AG, Basel

\section{Introduction}

Early diagnosis of melanoma relies on consumers' conduct of regular skin self-examination (SSE), vigilance to any changes on their skin and presenting to their medical practitioner if they notice any changes. Barriers to SSE include forgetfulness, lack of concern about mela-

This article is part of the Nevi Article Series

\section{KARGER}

(c) 2018 S. Karger AG, Basel

E-Mail karger@karger.com

www.karger.com/drm
Monika Janda

Centre for Health Services Research, Faculty of Medicine, The University of Queensland Building 33, Princess Alexandra Hospital Campus

Woolloongabba, QLD 4102 (Australia)

E-Mail m.janda@uq.edu.au 
noma and uncertainty of which skin lesions are suspicious $[1,2]$. Barriers to presenting to a medical practitioner promptly with lesions of concern include poor knowledge of melanoma, self-perceived low risk of melanoma, and time constraints and travel, particularly for rural or remote populations [3-8]. These barriers may delay an early diagnosis and could have a detrimental effect on the patient's prognosis. Mobile health applications (apps) may help facilitate SSE and enable consumers to play a much more active role in managing their health $[9,10]$.

Mobile teledermoscopy is a promising technology for engaging people in early detection and management of melanoma. Mobile teledermoscopy uses a smartphone, an app to manage images, and an attachable dermatoscope to magnify and photograph skin lesions to remotely send to a dermatologist $[10,11]$. These devices can be utilised as an efficient, low-cost communication tool between medical practitioners [12]. For example, a general practitioner can send images to a dermatologist for referral, or a nurse could image lesions as part of a triage system to reduce specialist waiting times and avoid substantial delays for urgent cases [13]. Alternatively, patients could detect their own suspicious lesions for assessment during SSE and communicate directly with their medical practitioner or monitor lesions identified by a medical practitioner.

Mobile teledermoscopy has shown comparable diagnostic accuracy and agreement to face-to-face diagnoses when conducted by medical practitioners [14] and consumers [15]. Using 3,021 images of skin lesions, Warshaw et al. [14] reported that the diagnostic agreement between a clinic dermatologist and teledermatologist was fair to substantial for primary diagnoses $(45.7-80.1 \% ; \kappa=0.32-$ 0.62 ). Manahan et al. [15] trialled patient-performed mobile teledermoscopy in 49 participants and found that there was a high sensitivity for at-risk lesions in the participant-level analysis (82\%), with reduced sensitivity in the lesion-level analysis (42\%). The participant-level analysis assumed that a person with at least 1 suspicious lesion would see a medical practitioner for a whole-body clinical skin examination. Further improvements are required when analysing results on a per-lesion basis, as consumers did not photograph some potentially concerning lesions, although none were suspicious of melanoma [15]. Wu et al. [16] showed that patient-led monitoring of skin lesions was highly concordant with clinical decisions $(97 \%$; $\kappa=0.87)$.

Given these promising preliminary findings, the number of mobile health apps and devices advertised directly to consumers continues to increase. More evidence for

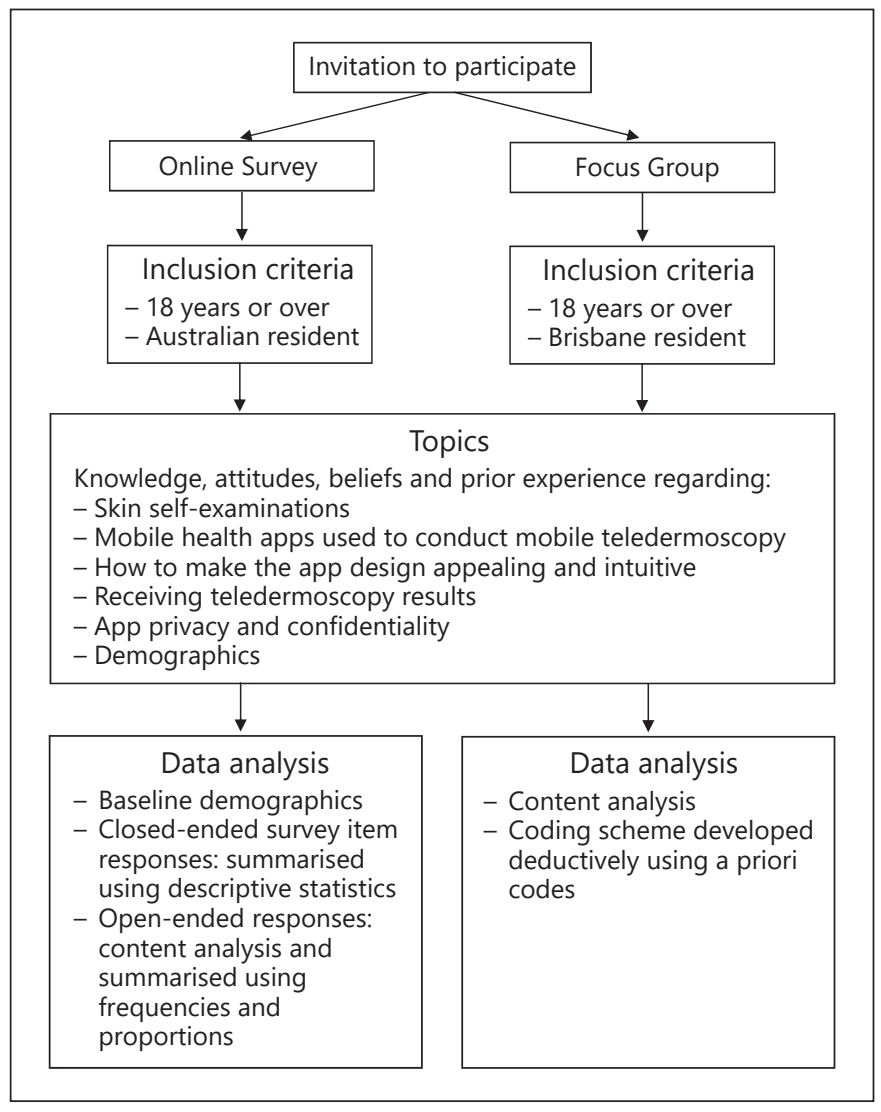

Fig. 1. Flowchart of Material and Methods.

their utility and understanding how to make these apps consumer-friendly and safe is required $[17,18]$. In this study, we conducted an online survey and focus groups to explore the factors that may influence consumers' adoption of a mobile health app to conduct mobile teledermoscopy including: (i) current SSE practices, (ii) acceptance of mobile teledermoscopy to support their skin checks, (iii) barriers to adopting mobile teledermoscopy and (iv) their thoughts on how the app should work including design and functionality. This work informed the building of a new consumer-directed app for conducting mobile teledermoscopy that will be tested in a randomised controlled trial.

\section{Material and Methods}

For further details, see the online supplementary material (for all online suppl. material, e.g. Materials and Methods and the corresponding Appendix, see www.karger.com/doi/10.1159/000493728) (Fig. 1) [19]. 


\section{Results}

\section{Demographics}

All 88 participants who started the survey completed all questions, and hence all were included in the analyses. Participants who completed the online survey had a mean age of 38 years (range: $19-65$ years), and 17 (19\%, 17/88) were male. Twenty-one $(24 \%, 21 / 88)$ of the online survey participants reported having had 1 or more melanoma(s) removed in the past.

A total of 28 participants took part in one of four focus groups (range: 4-11 participants per focus group). Compared to the online survey participants, the focus group participants were older with a mean age of 46 years (range: $22-69$ years) and a greater proportion were male $(61 \%$, $17 / 28)$. The age was missing for 8 focus group participants. Eight $(29 \%, 8 / 28)$ of the focus group participants reported having had 1 or more previous melanomas removed.

\section{Skin Self-Examinations}

Regular SSEs were conducted by $56(64 \%, 56 / 88)$ of the online survey participants. Some of the reasons for conducting regular SSEs included: awareness of being at melanoma risk $(46 \%, 26 / 56)$ and personal $(18 \%, 10 / 56)$ or family history $(30 \%, 17 / 56)$ of melanoma. Participants who reported being aware of their melanoma risk were vigilant in checking their skin due to their high-risk phenotype, such as fair skin and personal history of melanoma. Participants who had a personal history of melanoma were concerned about re-occurrences of melanoma: "I am concerned about getting others [melanomas] again" (respondent 2) and "worried that I will get another [melanoma]" (respondent 35). Participants who had a family history of melanoma were often reminded by their family members to check their skin. Other reasons for conducting regular SSEs were being exposed to the harsh Australian environment $(11 \%, 6 / 56)$ and high exposure to ultraviolet radiation in the past $(11 \%, 6 / 56)$.

Thirty-two $(36 \%, 32 / 88)$ of the online survey participants did not regularly check their skin. The main reasons for not performing SSEs were forgetfulness $(44 \%, 14 / 32)$, perceived low risk of melanoma $(25 \%, 8 / 32)$ and not being confident in conducting SSEs $(25 \%, 8 / 32)$. Individuals who did not conduct SSEs reported they "never felt a serious risk of melanoma" (respondent 70) and were "never shown how or instructed to by my physician" (respondent 69). Some of the participants reported that they did not conduct SSE because they preferred to rely on a medical practitioner to check $(9 \%, 3 / 32)$.
The focus group participants expressed similar reasons for conducting or not conducting regular SSEs as the online survey respondents. Most participants were aware and pro-active about their melanoma risk. The majority of participants had high pre-existing knowledge of the importance of skin checks. Reasons for being pro-active about their SSEs included mindfulness of the high ultraviolet radiation in Australia, having a phenotype at higher risk of melanoma and/or a personal/family melanoma history.

Focus group participants who did not regularly conduct SSEs provided the following reasons: not confident in checking their own skin, lack of education on which skin lesions were suspicious, laziness, not being able to see all areas of their body and having to rely on a partner to check these hard-to-see areas. Participants expressed that conducting regular SSE was "not something that was in the forefront of (their) mind" and would only check "when worried." One participant mentioned that if he found a suspicious skin lesion during an SSE, then he would delay having a medical practitioner assess the lesion until other medical concerns became evident. In each of the four focus groups, participants preferred to rely on face-to-face skin examinations by a medical practitioner, rather than conducting regular SSEs, as they "don't know how to tell if a mole is bad."

\section{Mobile Teledermoscopy}

The vast majority of 88 online survey participants (95\%, 84/88) would consider sending photos of their skin lesions to a medical practitioner via a mobile health app. After watching the app demonstration video, participants were asked to list the advantages of the app. Advantages mentioned included the app was user-friendly $(32 \%$, $28 / 88)$ and convenient $(73 \%, 64 / 88)$, as it would be a "quick and easy way of determining whether or not an inperson appointment is necessary" (respondent 62) (Table 1). Participants also liked the idea of mobile teledermoscopy as it would reduce worry $(11 \%, 10 / 88)$, reduce cost of seeing a dermatologist $(8 \%, 7 / 88)$ and minimise embarrassment from undressing in front of a medical practitioner $(6 \%, 5 / 88)$. Some participants indicated that they would like to use mobile teledermoscopy to receive a second opinion $(6 \%, 5 / 88)$ or to monitor changes in their moles $(5 \%, 4 / 88)$, as it would "be a great way to objectively monitor a lesion that appears to be changing" (respondent 37$)$.

Four $(5 \%, 4 / 88)$ online survey participants would not consider using mobile teledermoscopy because they be- 
Table 1. Advantages and disadvantages of mobile teledermoscopy as perceived by online survey participants $(n=88)$

\begin{tabular}{|c|c|}
\hline Advantages & Disadvantages \\
\hline $\begin{array}{l}\text { Perceived usefulness } \\
\text { - Provide convenience for self-monitoring of skin lesions } \\
\quad(73 \%, n=64) \\
\text { - Maintain records }(14 \%, n=12) \\
\text { - Monitor changes in skin lesions }(14 \%, n=12) \\
\text { - Reduce cost of seeing a dermatologist in person } \\
\quad(13 \%, n=11) \\
\text { - Enhance examination in rural populations } \\
\quad(13 \%, n=11) \\
\text { - Improve early detection of melanoma }(8 \%, n=7) \\
\text { - Improve skin awareness }(6 \%, n=5) \\
\text { - Increase access to specialist advice }(5 \%, n=4) \\
\text { - Reduce concerns about melanoma diagnosis } \\
\quad \text { (9\%, } n=8) \\
\text { Perceived ease of use } \\
\text { - User-friendliness ( } 32 \%, n=28) \\
\text { - More private to photograph at home }(7 \%, n=6)\end{array}$ & $\begin{array}{l}\text { Perceived usefulness } \\
\text { - Increase cost and accessibility of mobile dermatoscopes }(15 \%, n=13) \\
\text { - Reduce accuracy of diagnoses as medical practitioners are unable to } \\
\text { palpate lesion }(5 \%, n=4) \\
\text { Perceived ease of use } \\
\text { - Hard to reach body areas }(15 \%, n=13) \\
\text { - Lack of education to select suspicious lesions }(11 \%, n=10) \\
\text { - Poor functionality }(6 \%, n=5) \\
\text { - Reduce phone memory to store app and images }(3 \%, n=3) \\
\text { Attitudes towards use } \\
\text { - Increase anxiety from self-monitoring using this technology } \\
\quad(13 \%, n=11) \\
\text { - Impact negatively on medical practitioner-patient relationships } \\
\quad(13 \%, n=11) \\
\text { - Increase privacy and confidentiality concerns }(25 \%, n=22) \\
\text { - Reduce trust in telediagnosis if diagnosis is not accurate }(5 \%, n=4)\end{array}$ \\
\hline
\end{tabular}

lieved mobile teledermoscopy would not be as accurate as a face-to-face consultation.

The idea of mobile teledermoscopy was also well accepted by most of the focus group participants. The focus group participants thought that mobile teledermoscopy would be convenient for monitoring skin spots over time and for consulting with their medical practitioner between face-to-face visits. Furthermore, participants thought mobile teledermoscopy would save time and money. The focus group participants expressed that mobile teledermoscopy would be beneficial for those residing in rural areas or who have mobility issues which would impact their ability to travel. Overall, these participants thought the app presented to them in this study was intuitive and was appealing, particularly for younger generations.

The focus group participants also identified barriers to mobile teledermoscopy. Barriers relevant to the medical practitioners included: the potential for overloading the system with many images, accuracy of image diagnoses, extra workload for medical practitioners and setting an affordable price to use the service. The concerns for the participants were that the app could increase anxiety while waiting for a response from the teledermatologist and whether medical providers could meet an acceptable timeline of a few hours to 1 week for a telediagnosis. Participants also acknowledged it would be difficult for the consumer to know which spots to photograph. One par-

Consumer Acceptance of a Mobile Health Application ticipant suggested the technology would be best utilised by medical practitioners who supervised the skin lesion monitoring process stating, "I think those kinds of apps first should be used as communication facilitators among health care professionals and patients, rather than being a diagnostic tool."

\section{App Functionality and Design}

Important features of the mobile teledermoscopy app included: instructions on how to take a good-quality image, example images of what constitutes a suspicious lesion and directions on which image to monitor from a medical practitioner. Participants would like to receive reminders to conduct SSEs via push notifications. To be acceptable, the image-taking and sending process needs to be quick and uncomplicated.

From the focus groups, participants reported they would not use apps that took a long time to load, required too many updates or required a large amount of phone memory. Any such features could result in the user deleting the app from their smartphone. They would like the app to integrate single sign-on using their existing information from a social media service, thus removing the step of entering personal details to set up an account. App transparency was important, and participants would like to see reputable organisations and dermatologist's credentials endorsing the service. Participants stated that these endorsements would improve trust in the service. 


\section{Privacy and Confidentiality}

Twenty-two $(25 \%, 22 / 88)$ online survey participants were concerned about privacy and confidentiality of sending images of their skin lesions via the app. Reasons for privacy and confidentiality concerns included: possibility of online hacking $(27 \%, 6 / 22)$, photographs of skin lesions in sensitive body areas $(9 \%, 2 / 22)$ and misuse of data $(9 \%, 2 / 22)$. Sixty-six $(75 \%, 66 / 88)$ online participants who were not worried about privacy and confidentiality issues felt that the benefits of mobile teledermoscopy would outweigh the risks of privacy breaches and were not concerned to send skin lesion images as a "picture of a spot is not revealing" (respondent 78).

Focus group participants were similarly divided about privacy and confidentiality concerns. Some participants expressed concerns regarding health insurance companies obtaining their data and the possibility of hacking. The majority of participants were not concerned about privacy and confidentiality issues surrounding sending of images. Participants were more concerned about the personal medical history that accompanied the images.

\section{Discussion}

This study is one of the first to focus on consumers' views about conducting mobile teledermoscopy in the home environment to accompany SSE and found that the idea of using mobile teledermoscopy was generally well accepted. Little is known about consumers' views of mobile teledermoscopy and the factors that may affect consumers' adoption of this technology. Most previous studies that explored acceptance of mobile teledermoscopy focused on clinician acceptance [5, 19-27].

\section{Skin Self-Examinations}

In our study, $64 \%$ of survey participants reported conducting regular SSEs. This was higher than the prevalence of SSE (55\%) assessed in a cross-sectional study of 40,172 adults living in Queensland, Australia [28]. One of the main reasons reported in our study for why people did not conduct regular SSEs was because they were not confident in deciding which skin lesions were suspicious. This was also a common theme identified in each of the four focus groups conducted. In our study, participants perceived that an app incorporating SSE instructions would improve their confidence to conduct SSE.

\section{Mobile Teledermoscopy}

Overall, consumers perceived that mobile teledermoscopy could be beneficial to help them monitor their skin lesions and enhance earlier detection of melanoma. Perceived usefulness and perceived ease of use are constructs that are integral to the adoption of technology $[23,29]$. Our results were similar to two previous studies that assessed the acceptance of consumer-driven mobile teledermoscopy $[9,25]$. The first study assessed the acceptance of conducting mobile teledermoscopy in the home environment for the early detection of melanoma $(n=228)$. Prior to conducting mobile teledermoscopy, 91\% agreed that they would find it beneficial [9]. This study also assessed acceptance after use in a subsample of participants who actually used teledermoscopy $(n=$ 49 ) and found $94 \%$ felt it was easy to conduct and $86 \%$ reported it would encourage more frequent skin checks. In the second study, a small pilot group of 10 participants found the dermatoscope easy to use [10]. In another study, Marchell et al. [25] conducted both face-to-face $(n=206)$ and teledermoscopy $(n=209)$ performed by a medical practitioner and found that participants preferred face-to-face over teledermoscopy. However, overall satisfaction with teledermatology was still high. Participants provided an overall satisfaction score of 4.74 for teledermatology on a 5-point Likert scale with 5 indicating greatest satisfaction [25]. The main concern that could affect attitudes towards the use of mobile teledermoscopy was the reliability of the telediagnoses. Horsham et al. [9] found $45 \%$ of 228 participants would be concerned about the accuracy of the telediagnosis. The feedback received from this study will guide the updates of the mobile health app, which will be tested in a randomised controlled trial.

In this study, some participants reported that mobile teledermoscopy would reduce the cost of seeing a dermatologist. A previous review reported people would be willing to pay out of pocket expenses for access to teledermatology services [30]. Spinks et al. [31] conducted a discrete choice experiment to investigate consumer preferences for melanoma screening options using different health services compared to SSE. These investigators determined that participants would be willing to pay up to AUD 110 for mobile teledermoscopy with a dermatologist reviewing the images [31]. The evidence suggests consumers are willing to adopt the technology, but further research is required to determine the accuracy of mobile teledermoscopy compared to face-to-face diagnosis [12]. 


\section{Privacy and Confidentiality}

Privacy and confidentiality are important to consider when developing a mobile health app as it may contain sensitive images. The majority of participants were not concerned about privacy and confidentiality issues as they felt that images of skin lesions cannot identify them. However, some participants did express concerns. One concern was the possibility of third parties gaining access to their personal information, in particular private health insurance companies who may use this information to increase premiums or decrease health insurance cover. Wu et al. [16] assessed patient acceptance of teledermoscopy for short-term monitoring of pigmented lesions $(n=29)$ and found the opposite, that increased privacy and comfort level was one of the most frequently cited reasons for use of teledermoscopy.

\section{Strengths and Limitations}

Strengths of the study include using both a qualitative and quantitative approach to better understand people's views on barriers and enablers of mobile teledermoscopy. The study was limited by the small sample size and the voluntary nature of the participant sample as it is likely that those who responded to the study invitation were more interested in melanoma early detection and had strong viewpoints on mobile teledermoscopy compared to the general population; hence, the results may not be generalisable to the general population. Furthermore, the participants were unable to experiment with the mobile health application and mobile dermatoscope, which could have hindered their judgment of the technology.

\section{Conclusion}

Consumers support the use of mobile teledermoscopy as an initial point of contact and adjunct to face-to-face dermatology consultations. Mobile teledermoscopy may be a useful tool to complement SSE meriting further study on the accuracy of telediagnoses and translation into clinical practice.

\section{Key Message}

Consumers accepted the use of mobile teledermoscopy to improve the detection of melanoma.

\section{Acknowledgements}

The authors would like to thank Linda Finch for her contribution to the research. This project is being undertaken as part of a research project on behalf of Queensland University of Technology and the University of Queensland, in partnership with FotoFinder PTY, the Princess Alexandra Hospital Foundation, Melanoma Patients Australia, the Skin and Cancer Foundations (VIC, NSW, QLD), and the Dermatology Departments of the University of Graz, the University of Arizona and Memorial Sloan Kettering Cancer Center, USA.

\section{Statement of Ethics}

This study was approved by the Queensland University of Technology Human Ethics Committee (QUT approval No. 1400000807).

\section{Disclosure Statement}

H. Peter Soyer is a shareholder of e-derm consult $\mathrm{GmbH}$ and MoleMap by Dermatologists Ltd Pty. He provides teledermatological reports regularly for both companies. He is also advisor of First Derm ${ }^{\mathrm{TM}}$.

\section{Funding Sources}

The study is funded by a research grant awarded to M.J. from the National Health and Medical Research Council APP1113962 and APP1099021. M.J. is funded by a TRIP Fellowship APP1151021. H.P.S. is also funded by the Medical Research Future Fund - Next Generation Clinical Researcher's Program Practitioner Fellowship (APP1137127).

\section{Author Contributions}

All authors contributed significantly to the design of the research study and interpretation of the data. U.K., C.H. and M.J. participated in the drafting of the work, and all authors critically revised it. All authors have reviewed and approved the final version for submission.

\section{References}

1 Janda M, Youl PH, Lowe JB, Baade PD, Elwood M, Ring IT, et al. What motivates men age $[\{G T\}]$ or $=50$ years to participate in a screening program for melanoma? Cancer. 2006 Aug; 107(4):815-23.

2 Janda M, Youl PH, Lowe JB, Elwood M, Ring IT, Aitken JF. Attitudes and intentions in relation to skin checks for early signs of skin cancer. Prev Med. 2004 Jul;39(1):11-8. 
3 Sankaranarayanan J, Sallach RE. Rural patients' access to mobile phones and willingness to receive mobile phone-based pharmacy and other health technology services: A pilot study. Telemed J E Health. 2014;20(2):182-5.

4 Mehrotra A, Huskamp HA, Souza J, UscherPines L, Rose S, Landon BE, et al. Rapid Growth In Mental Health Telemedicine Use Among Rural Medicare Beneficiaries, Wide Variation Across States. Health Aff (Millwood). 2017 May;36(5):909-17.

5 Durupt M, Bouchy O, Christophe S, Kivits J, Boivin JM. Telemedicine in rural areas: General practitioners' representations and experiences. Santé Publ. 2016 Oct;28(4):487-97.

6 Nelson R. Telemedicine and Telehealth: The Potential to Improve Rural Access to Care. Am J Nurs. 2017 Jun;117(6):17-8.

7 Swetter SM, Johnson TM, Miller DR, Layton CJ, Brooks KR, Geller AC. Melanoma in middle-aged and older men: a multi-institutional survey study of factors related to tumor thickness. Arch Dermatol. 2009 Apr;145(4):397404.

8 Williams HA, Fritschi L, Beauchamp C, Katris P. Evaluating the usefulness of self-reported risk factors in a skin cancer screening program. Melanoma Res. 2006 Aug; 16(4):341-5.

9 Horsham C, Loescher LJ, Whiteman DC, Soyer HP, Janda M. Consumer acceptance of patient-performed mobile teledermoscopy for the early detection of melanoma. Br J Dermatol. 2016 Dec;175(6):1301-10.

10 Janda M, Loescher LJ, Soyer HP. Enhanced skin self-examination: a novel approach to skin cancer monitoring and follow-up. JAMA Dermatol. 2013 Feb;149(2):231-6.

11 Massone C, Hofmann-Wellenhof R, Ahlgrimm-Siess V, Gabler G, Ebner C, Soyer HP. Melanoma screening with cellular phones. PLoS One. 2007 May;2(5):e483.
12 Snoswell CL, Caffery LJ, Whitty JA, Soyer HP, Gordon LG. Cost-effectiveness of Skin Cancer Referral and Consultation Using Teledermoscopy in Australia. JAMA Dermatol. 2018 Jun;154(6):694-700.

13 Lim D, Oakley AM, Rademaker M. Better, sooner, more convenient: a successful teledermoscopy service. Australas J Dermatol. 2012 Feb;53(1):22-5.

14 Warshaw EM, Gravely AA, Nelson DB. Reliability of store and forward teledermatology for skin neoplasms. J Am Acad Dermatol. 2015 Mar;72(3):426-35.

15 Manahan MN, Soyer HP, Loescher LJ, Horsham C, Vagenas D, Whiteman DC, et al. A pilot trial of mobile, patient-performed teledermoscopy. Br J Dermatol. 2015 Apr;172(4): 1072-80.

16 Wu X, Oliveria SA, Yagerman S, Chen L, DeFazio J, Braun R, et al. Feasibility and Efficacy of Patient-Initiated Mobile Teledermoscopy for Short-term Monitoring of Clinically Atypical Nevi. JAMA Dermatol. 2015 May;151(5): 489-96.

17 McCurdie T, Taneva S, Casselman M, Yeung M, McDaniel C, Ho W, et al. mHealth consumer apps: the case for user-centered design. Biomed Instrum Technol. 2012;46(2 Suppl): 49-56.

18 Abbott LM, Smith SD. Smartphone apps for skin cancer diagnosis: implications for patients and practitioners. Australas J Dermatol. 2018 Aug;59(3):168-70.

19 Abbott LM, Magnusson RS, Gibbs E, Smith SD. Smartphone use in dermatology for clinical photography and consultation: Current practice and the law. Australas J Dermatol. 2018 May;59(2):101-7.

20 Chao JT 2nd, Loescher LJ, Soyer HP, CurielLewandrowski C. Barriers to mobile teledermoscopy in primary care. J Am Acad Dermatol. 2013 Nov;69(5):821-4.

21 Mehrtens SH, Halpern SM. Changing use and attitudes towards teledermatology in the UK over 10 years: Results of the 2016 National Survey. Br J Dermatol. 2018 Jan;178(1):2868.
22 Wade VA, Eliott JA, Hiller JE. Clinician acceptance is the key factor for sustainable telehealth services. Qual Health Res. 2014 May; 24(5):682-94.

23 Orruño E, Gagnon MP, Asua J, Ben Abdeljelil A. Evaluation of teledermatology adoption by health-care professionals using a modified Technology Acceptance Model. J Telemed Telecare. 2011;17(6):303-7.

24 Green TH, Gillespie N. Service provider's experiences of service separation: The case of telehealth. J Serv Res. 2016;19(4):477-94.

25 Marchell R, Locatis C, Burgess G, Maisiak R, Liu WL, Ackerman M. Patient and provider satisfaction with teledermatology. Telemed J E Health. 2017 Aug;23(8):684-90.

26 Stronge AJ, Nichols T, Rogers WA, Fisk AD. Systematic human factors evaluation of a teledermatology system within the US military. Telemed J E Health. 2008;14(1):25-34.

27 Stratton D, Loescher LJ. The acceptance of mobile teledermoscopy by primary care nurse practitioners in the state of Arizona. J Am Assoc Nurse Pract. 2016 Jun;28(6):287-93.

28 Olsen CM, Neale RE, Green AC, Webb PM, Whiteman DC; The QSkin Study; The Epigene Study. Independent validation of six melanoma risk prediction models. J Invest Dermatol. 2015 May;135(5):1377-84.

29 Davis FD. Perceived usefulness, perceived ease of use, and user acceptance of information technology. Manage Inf Syst Q. 1989; 13(3):319-40.

30 Finnane A, Dallest K, Janda M, Soyer HP. Teledermatology for the Diagnosis and Management of Skin Cancer: A Systematic Review. JAMA Dermatol. 2017 Mar;153(3):31927.

31 Spinks J, Janda M, Soyer HP, Whitty JA. Consumer preferences for teledermoscopy screening to detect melanoma early. JTelemed Telecare. 2016 Jan;22(1):39-46. 